\title{
Current understanding of SPEM and its standing in the preneoplastic process
}

\author{
Victoria G. Weis ${ }^{1}$ and James R. Goldenring ${ }^{1,2}$ \\ ${ }^{1}$ Departments of Surgery and Cell and Developmental Biology, Epithelial Biology Center, Vanderbilt University School of Medicine, \\ 10435G MRB IV, 2213 Garland Avenue, Nashville, TN 37232, USA \\ ${ }^{2}$ Nashville Department of Veterans Affairs Medical Center, Nashville, TN, USA
}

\begin{abstract}
Gastric cancer is the second leading cause of cancer-related death worldwide, but the details of gastric carcinogenesis remain unclear. In humans, two preneoplastic metaplasias are associated with the precancerous stomach: intestinal metaplasia and spasmolytic polypeptide-expressing metaplasia (SPEM). While mouse models of Helicobacter sp. infection have not shown intestinal metaplasia, a number of mouse models lead to the evolution of SPEM. In this review, we summarize increasing data that indicates that SPEM arises in the setting of parietal cell loss, either following acute druginduced oxyntic atrophy or in chronic oxyntic atrophy associated with $\boldsymbol{H}$. felis infection. Importantly, recent investigations support the origin of SPEM through transdifferentiation from mature chief cells following parietal cell loss. Novel biomarkers of SPEM, such as HE4, hold promise as specific markers of the metaplastic process distinct from normal gastric lineages. Staining with HE4 in humans and other studies in gerbils suggest that SPEM arises initially in the human stomach following parietal cell loss and then further evolves into intestinal metaplasia, likely in association with chronic inflammation. Further studies are needed to broaden our knowledge of metaplasia and early cancer-specific biomarkers that could give insights into both lineage derivation and preneoplasia detection.
\end{abstract}

Key words Gastric adenocarcinoma - Metaplasia - SPEM · Intestinal metaplasia

\section{Introduction}

Gastric adenocarcinoma is the second leading cause of cancer-related death worldwide [1]. Gastric resection remains the critical step in gastric cancer therapy. Accordingly, the early detection and resection of early-

Offprint requests to: J.R. Goldenring

Received: September 28, 2009 / Accepted: October 11, 2009 stage cancers remains a priority. However, the cellular mechanisms involved in the progression of preneoplastic events to gastric cancer remain unclear. This lack of a clear understanding of the neoplastic transition hampers efforts to discover better screening methods for populations at risk. Thus, a more extensive knowledge of the preneoplastic events could clearly reduce gastric cancer mortality. In the following discussion we will review emerging concepts on the origin of preneoplastic metaplasia and the factors that influence conversion of metaplasia towards neoplasia.

Oxyntic atrophy, metaplasia, and gastric adenocarcinoma

Chronic infection of the stomach with the gramnegative bacterium, Helicobacter pylori, is the leading proximate cause of gastric cancer in humans [2]. Chronic $H$. pylori infection causes global changes in the gastric mucosa which can eventually lead to gastric adenocarcinoma. These global changes result from the two major effects of chronic $H$. pylori infection: parietal cell loss (or oxyntic atrophy) and prominent inflammation. Oxyntic atrophy has a profound effect on the gastric mucosa because parietal cells play an important role in the differentiation of other gastric lineages. Parietal cells are responsible for the secretion of a number of factors, including amphiregulin, transforming growth factor (TGF)- $\alpha$, heparin binding-epidermal growth factor-like growth factor (HB-EGF), and sonic hedgehog (Shh) [3-6]. Loss of parietal cell-derived signaling molecules disrupts the proper differentiation of other lineages such as the zymogen-secreting chief cells [7]. The second major result of chronic infection is prominent inflammation throughout the mucosa. Oxyntic atrophy along with prominent inflammation appear to be the prerequisites for progression to metaplasia and gastric adenocarcinoma [8]. 
Oxyntic atrophy in the setting of inflammation can progress to the development of metaplasia. In humans, two types of metaplasia can arise from the presence of oxyntic atrophy and inflammation: intestinal metaplasia and spasmolytic polypeptide-expressing metaplasia (SPEM). Both intestinal metaplasia and SPEM have been associated with the progression to intestinal-type gastric cancer [9-18]. Thus, a case can be made for oxyntic atrophy in association with prominent inflammation laying the groundwork for altered gastric lineages that are involved in the development of gastric cancer. However, the factors mediating the progression from oxyntic atrophy to gastric cancer remain unclear.

Intestinal metaplasia is characterized by the presence of intestinal goblet cells in the stomach [19]. Because intestinal goblet cells are not normally present in the stomach, intestinal metaplasia was originally proposed as the preneoplastic metaplasia leading to intestinaltype cancer [9]. Goblet cells in intestinal metaplasia express appropriate intestinal markers, including Muc2 and Trefoil factor 3 (TFF3) [20]. However, recent investigations have identified a second possible preneoplastic metaplasia, SPEM. The mucous metaplastic lineages in SPEM display morphological characteristics more typical of deep antral gland cells or Brunner's glands, with expression of Muc6 and Trefoil factor 2 (TFF2) [16]. Despite the similarity between SPEM and the deep antral glands, no gastrin cells are observed in these glands. Recent studies have suggested that both SPEM and intestinal metaplasia may be preneoplastic metaplasias. In three separate studies, SPEM was associated with $90 \%$ of resected gastric cancers [16, 18, 21]. The exact influences promoting the sequential progression from oxyntic atrophy to metaplasia to dysplasia, however, remain unknown.

\section{Parietal cell loss leads to metaplasia}

The unresolved questions about the origin of oxyntic atrophy and its progression to gastric cancer are difficult to examine in humans, but mouse models are more amenable to answering these questions. As mentioned above, the most common predisposing factor for gastric cancer in humans is chronic H. pylori infections, resulting in oxyntic atrophy and prominent inflammation. The importance of both oxyntic atrophy and the inflammatory response has been examined in mice. Chronic infection of mice with $H$. pylori or with a different, but related, subspecies of Helicobacter felis results in profound loss of parietal cells and inflammation throughout the mucosa, similar to that seen in humans [22-24]. $H$. felis-infected C57BL/6 mice develop SPEM after 6 months of infection, demonstrating that oxyntic atrophy and inflammation can also lead to SPEM in mice. After
12 months of infection, the mice progress to gastritis cystica profunda, a dysplastic process in the fundic mucosa [22]. Importantly, however, the infected mice never develop intestinal metaplasia, suggesting that SPEM is the preneoplastic metaplasia in mice. The direct origin of SPEM, however, is difficult to elucidate from this chronic model complicated by the presence of both of the major predisposing factors: oxyntic atrophy and prominent inflammation.

To isolate the role of oxyntic atrophy from the role of inflammation in the development of SPEM, administration of pharmaceutical designation (DMP)-777 has been utilized to examine the role of acute oxyntic atrophy. DMP-777 is a cell-permeant neutrophil elastase inhibitor that ablates parietal cells without inciting an inflammatory response. Loss of parietal cells is due to the secondary activity of DMP-777 as a parietal cellspecific protonophore. After 3 days of DMP-777 administration, mice develop oxyntic atrophy. The oxyntic atrophy gives rise to SPEM after 10 to 14 days of DMP777 administration in the absence of inflammation [25]. Thus, SPEM develops as a direct result of the loss of parietal cells. However, DMP-777-induced SPEM never progresses to dysplasia even after a year of administration [26]. These findings demonstrate the important role of inflammation in the progression to gastric neoplasia. Immunodeficient mice infected with $\mathrm{H}$. felis do not develop oxyntic atrophy and thus do not develop SPEM or progress to dysplasia [27]. Therefore, in the case of pharmacological ablation of parietal cells, no inflammation is needed for parietal cell loss. More importantly, the parietal cell loss is sufficient for the development of SPEM.

\section{Cellular origin of metaplasia}

Although oxyntic atrophy leads to the emergence of SPEM, the cellular origin of SPEM has remained elusive. Normal gastric cell lineages such as the parietal cell and mucous neck cell are derived from progenitor cells located in the upper region of the gland [28-32]. As the post-mitotic mucous neck cells migrate towards the base of the gland, they alter their expression profiles as well as their morphology to redifferentiate into chief cells (Fig. 1) [30, 33]. Because both mucous neck cells and chief cells have conventionally been thought of as post-mitotic, previously we had suggested that SPEM cells arise from a cryptic progenitor cell located at the base of the gastric glands. However, recent studies have suggested that, in fact, the chief cells (or a subset of chief cells) may transdifferentiate into SPEM cells [25, $34,35]$. Indeed, the most reliable pattern for the emergence of SPEM following induced oxyntic atrophy has been the identification of cells at the bases of fundic 


\section{Normal Gland}

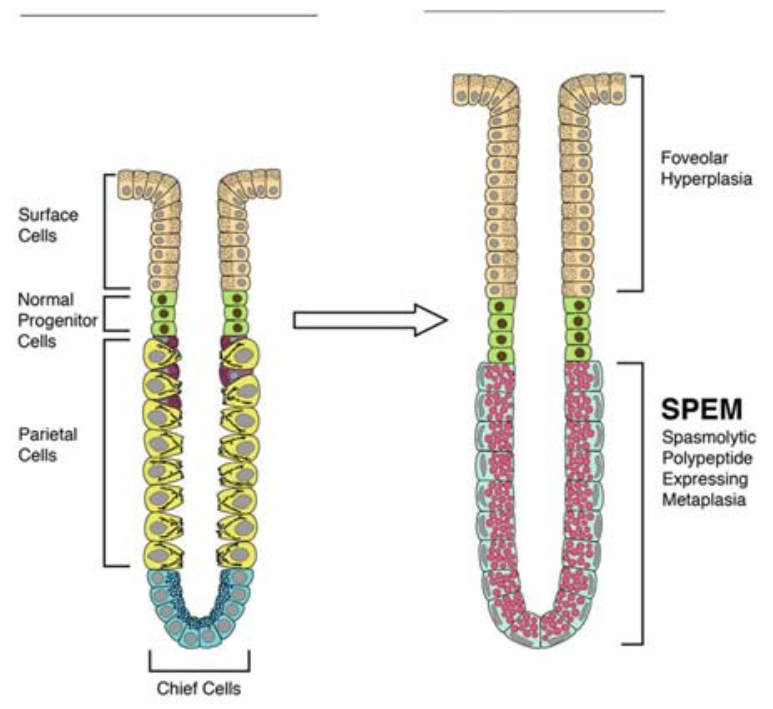

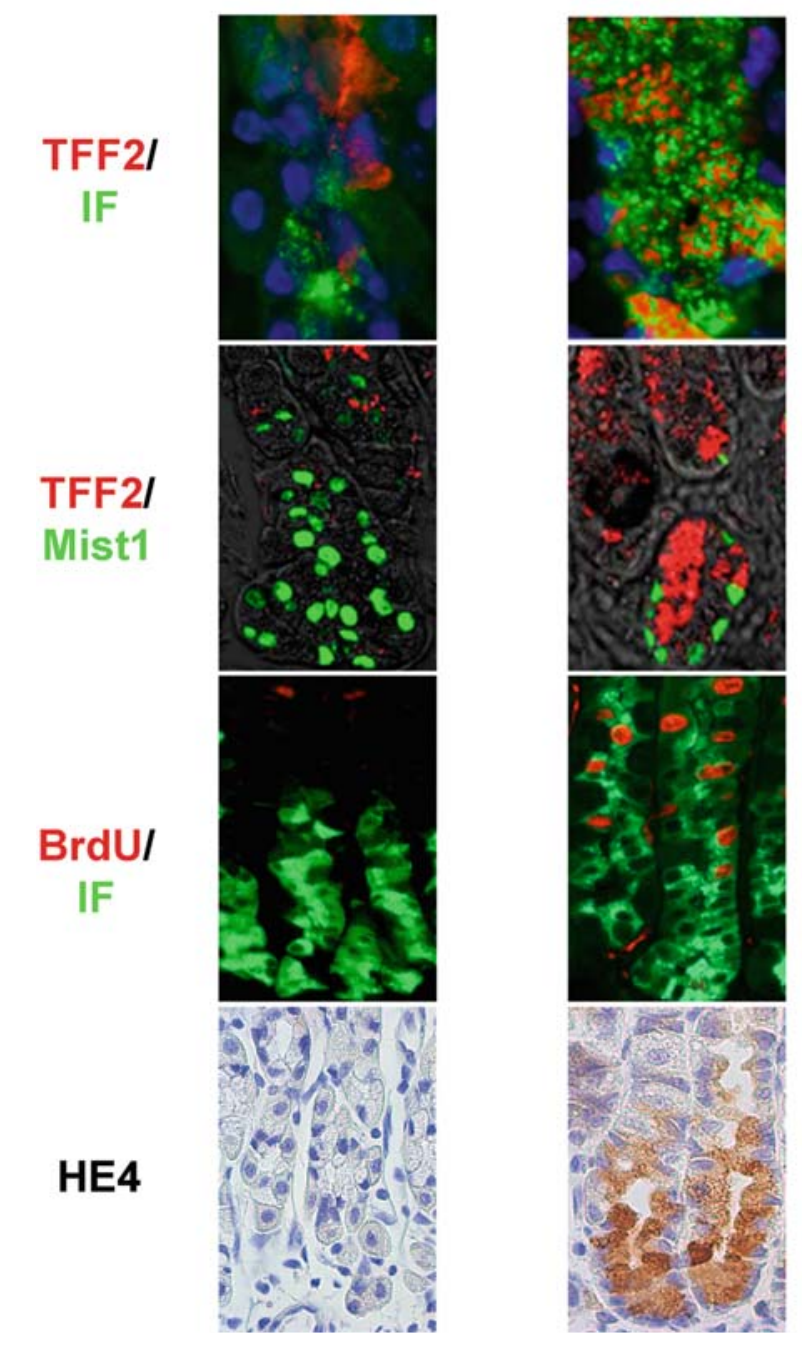

Fig. 1. Comparison of normal fundic gastric glands and metaplastic spasmolytic polypeptide-expressing metaplasia (SPEM) glands in mice. The diagram at the top depicts alterations in gland lineages between normal and SPEM glands with emergence of SPEM and foveolar hyperplasia following loss of parietal cells. The panels below show immunostaining patterns for normal and SPEM-containing glands. Trefoil factor 2 (TFF2; red)-expressing mucous neck cells redifferentiate into intrinsic factor (green)-expressing chief cells at the base of normal glands. However, TFF2 expression is expanded to the base of SPEM glands and appears within cells that show dual staining for intrinsic factor. Mist1 (green) is a differentiated chief cell marker in normal gastric glands. However, Mist1 is also expressed in some TFF2-expressing SPEM cells, suggesting transdifferentiation of chief cells. Although proliferation as detected by bromodeoxyuridine $(B r d U$; red) is normally shown only in the progenitor zone of the upper normal gland, TFF2-expressing SPEM cells show clear proliferating cells also expressing intrinsic factor $(I F)$. Previous studies have led to the identification of promising biomarkers of SPEM, such as HE4. HE4 is not detected in normal chief cells or any normal fundic cells, but HE4 staining is strongly observed in SPEM 
glands expressing both intrinsic factor (a mouse chief cell marker) and TFF2 in different granules within the same cells [25]. One recent study demonstrated that some SPEM cells express Mist1, a chief cell differentiation-specific marker [35]. Moreover, this same study showed that SPEM cells located towards the base of the gland have an increase in the expression of minichromosome maintenance (MCM)3, which is involved in the initiation of genome replication. Occasionally, MCM3-positive cells were also observed at the bases of normal gastric glands in cells that coexpressed intrinsic factor, a chief cell marker in mice, suggesting that a subset of chief cells may retain or possibly can reactivate the ability to replicate [35]. Future studies are needed to investigate further the possibility that SPEM arises from chief cells, and more specifically, from the MCM3-positive chief cells. Nevertheless, present information supports the origin of SPEM from cells distinct from the normal mucosal progenitor cells located in the gland neck.

The origin of intestinal metaplasia has been harder to elucidate because Helicobacter infection in mice does not result in intestinal metaplasia. However, $H$. pylori infection of other species, such as Mongolian gerbils, does induce intestinal metaplasia [36, 37]. Moreover, $H$. pylori infection-induced intestinal metaplasia evolves into gastric cancer in older Mongolian gerbils [38-40]. Detailed examination of the histological progression of oxyntic atrophy to gastric cancer in the Mongolian gerbil has shed some light into the origin of intestinal metaplasia and its association with SPEM [40]. After only 3 weeks of infection, the gerbils developed SPEM in the presence of oxyntic atrophy. The emergence of intestinal metaplasia followed later, at 24 and 39 weeks of infection. Importantly, the sites of intestinal metaplasia were surrounded by the preexisting SPEM. Moreover, single glands contained both intestinal metaplasia and SPEM [40]. Similar developmental patterns of intestinal metaplasia have also been observed in humans [41]. The findings in these studies suggest that oxyntic atrophy results in the development of SPEM, which, in turn can then evolve into intestinal metaplasia (Fig. 2). However, conclusive evidence of this progression has evaded researchers so far. Future studies in mouse models of SPEM and intestinal metaplasia are needed to trace this progression of metaplastic lineages. Until these studies are conducted, three hypotheses must be considered. First, each type of metaplasia (intestinal metaplasia or SPEM) may lead to a distinct type of cancer. For example, SPEM may progress to gastrictype cancers while intestinal metaplasia proceeds to intestinal-type cancers. Alternatively, one of the metaplasias could be the preneoplastic metaplasia, while the other is merely associated with cancer. The third hypothesis based on the Mongolian gerbil studies is that SPEM develops first, progresses to intestinal metaplasia, and finally evolves into gastric cancer. More extensive studies comparing both the transcript and protein expression patterns of metaplastic lineages to the patterns of gastric adenocarcinomas may provide insights into the links between metaplastic lineages and cancer.

Another aspect of this progression that must be considered, though it is not frequently mentioned, is the geographical origin of these metaplastic lesions within the stomach. SPEM and the resulting intestinal metaplasia found in $H$. pylori-infected Mongolian gerbils initiated along the lesser curvature of the stomach and expanded towards the greater curvature during longer terms of infections [40]. This concentration of metaplastic lineages along the lesser curvature has also been documented in humans [41, 42]. Additionally, H. pylori preferentially colonizes the lesser curvature both in mouse models and in humans [43, 44]. This localization of metaplastic initiation in both rodents and humans may be the result of Helicobacter sp. colonization patterns. However, there may also be an underlying factor for this convergence on the lesser curvature that has yet to be discovered. For example, the cellular lineage composition of the lesser curvature may vary from that of the greater curvature to account for these differences in the location of metaplastic initiation. Interestingly, the human stomach has not yet been extensively mapped out. It will be interesting to know the full extent of the differences in lineage distributions between the two curvatures and how they correspond with $H$. pylori colonization and the origin of metaplasia.

It is also necessary to consider the other geographical axis when examining the origin of metaplasias. Unlike the possible lineage differences between the two curvatures, there are known differences in the cell lineages between the fundus and the antrum of the stomach. Although parietal cells play a crucial signaling role in the fundus, they are not found in the antrum. Chief cells are also not located in the antrum. Instead, antral glands have a TFF2-expressing lineage that populates the base of the glands. The antrum also contains other cell lineages not found in the fundus, such as gastrinexpressing G cells. Gastrin plays an important role in acid secretion as well as proliferation in the fundus $[45$, 46]. Knocking out gastrin creates a hypogastrinemic environment which results in a reduction in parietal cell mass in the fundus and chronic inflammation in the antrum. This antral inflammation leads to an increase in intestinal markers such as villin and mucin (MUC)2, although goblet cell morphology is not seen. Eventually, tumors develop in the antrum in 12-month-old gastrin-deficient mice [47]. Interestingly, another mouse model, the TFF1 knockout mouse, also results in antral tumors, although more quickly. TFF1, unlike gastrin, is 

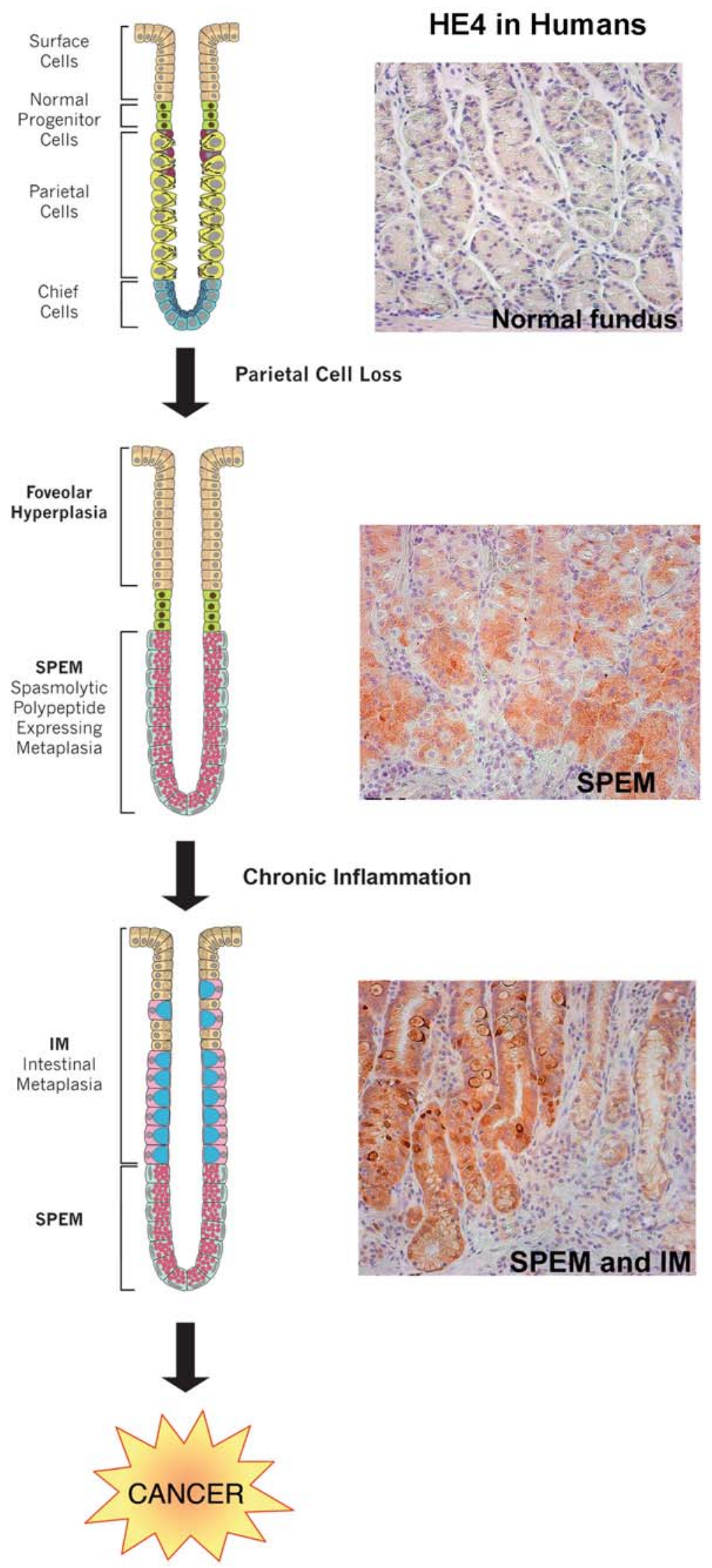

Fig. 2. Current model for the origin and progression of gastric metaplasias in humans. Studies have demonstrated that loss of parietal cells results in chief cell transdifferentiation and the emergence of SPEM. In the presence of chronic inflammation from Helicobacter pylori infection, SPEM evolves into intestinal metaplasia and then progresses on to gastric cancer. Establishment of biomarkers of these metaplastic lineages is a priority. Recent studies in mice identified HE4 as a SPEM biomarker. In humans, HE4 is not expressed in the cells of the normal fundus. However, HE4 is detected in both SPEM and intestinal metaplasia $(I M)$, supporting the hypothesis of SPEM progression to intestinal metaplasia 
normally expressed in surface cells throughout the entire stomach. Mice lacking TFF1 expression develop antral tumors at 5 months of age [48]. Both mouse models suggest an important role for gastrin and TFF1 in maintaining normal differentiation of the antrum. A third mouse model combines the effect of gastrin and TFF1 loss. The gp130 mouse contains a mutation that inhibits SHP2 binding to the interleukin (IL)-6 family coreceptor gp130. This binding inhibition results in decreases in gastrin and TFF1 expression, as well as an increase in signal transducer and activator of transcription (STAT) 3 expression. These aberrant expression patterns result in antral tumors at 6 weeks of age, which quickly extend into the fundus by around 17 weeks of age. Additionally, these tumors are accompanied by SPEM [49]. Thus, the combined effect of gastrin loss with TFF1 loss accelerates the development of antral tumors in mice. Moreover, this model resembles antral tumor development in humans. Together, gastric tumor models, in mice and Mongolian gerbils, demonstrate how metaplasias of the stomach and gastric cancer may originate and progress differently depending on the geographical location and possibly local regulatory signals.

\section{Regulation of metaplasia}

While oxyntic atrophy is directly responsible for the development of SPEM, the emergence of SPEM can be altered by endocrine or paracrine regulation. As stated above, parietal cells produce instructive signaling for the growth and differentiation of the other gastric lineages. However, parietal cells also receive signals from other cell types in the gastric mucosa, such as the previously mentioned gastrin-secreting $G$ cells and histamine-secreting enterochromaffin-like (ECL) cells [50-56]. Gastrin stimulates ECL cell release of histamine, the primary stimulator of acid secretion from parietal cells [51]. Histamine secreted by the ECL cells also plays an important role in the differentiation of the gastric lineages [57]. At the same time, somatostatin cells coordinate inhibitory influences both on acid secretion and hormonal secretion [58]. Thus, parietal cells, G cells, somatostatin cells, and ECL cells coordinate with each other to regulate the gastric mucosal milieu. Understandably then, the disruption of this balance of intramucosal factors affects the maturation of normal gastric lineages as well as the development of SPEM. For example, gastrin-deficient mice develop SPEM after only 1 day of DMP-777 administration, while it takes 10 days of DMP777 administration for the development of SPEM in wild-type mice [25]. Similarly, histidine-decarboxylase (HDC)-deficient mice (which lack histamine) also have an accelerated development of SPEM upon administration of DMP-777 [57]. These studies highlight the complex coordination that occurs between the different cell lineages of the stomach.

Additionally, other intramucosal paracrine factors such as growth factors are important in the regulation of metaplasia. To examine the role of the EGF signaling pathway in the development of SPEM, the waved-2 mouse was utilized, because it has a reduction in the EGF receptor tyrosine kinase activity. Upon administration of DMP-777, accelerated development of SPEM was observed in the waved-2 mice [59]. Further investigations into this signaling pathway demonstrated that different EGF receptor ligands affected the mucosal lineages differently. DMP-777-induced SPEM in TGF $\alpha$ deficient mice developed similarly to that seen in wild-type mice. In contrast, loss of amphiregulin led to accelerated SPEM development [60]. Taken together, these findings demonstrate that the emergence of SPEM is regulated by a complex coordination of both endocrine and paracrine factors. While loss of a single factor may alter SPEM development, no factor has been found to be solely responsible for the emergence of SPEM.

\section{Metaplastic progression to gastric cancer}

Inflammation plays an important role in the development and progression of SPEM. As stated before, while SPEM can develop in the absence of inflammation, it does not progress to dysplasia without inflammation. Helicobacter infections in mice progress to dysplasia when prominent inflammation is present [61]. The inflammatory response observed in these mice is composed mostly of a T-helper (Th)1 response [62]. Interestingly, further investigation into the components of inflammation has led to the discovery that the type of inflammatory response is important in the progression to gastric cancer. In mice with a Th2-dominant inflammatory response to $H$. felis infection, the SPEM did not progress to dysplasia [63]. Thus, a Th1-dominant inflammatory response is needed for the progression of SPEM to dysplasia in mice. Other factors involved in the progression to neoplasia have been difficult to elucidate, possibly because of gaps in our knowledge of the progression from metaplasia to dysplasia.

One recent study suggested a role for bone marrowderived cells (BMDCs) within this progression [64]. This study found that BMDCs were recruited to the stomach during chronic $H$. felis infection. These recruited BMDCs appeared to engraft into the SPEM glands and progress to gastritis cystica profunda [64]. No engraftment was observed in models of SPEM without inflammation, such as DMP-777 treatment. It also remains unclear whether BMDCs adopt the SPEM phenotype or actually fuse with SPEM cells during 
engraftment. At this time it remains uncertain whether this engraftment of SPEM by BMDCs is specific to infection with the $H$. felis strain, and there remains no strong evidence to support a role for BMDCs in the evolution of metaplasia or cancer in humans.

Another issue to consider is the concept of field cancerization and expansion of mutated clones. Field cancerization suggests that multiple stem cells form independent epithelial tumors [65]. Application of this concept to SPEM and intestinal metaplasia proposes that one of the stem cells in the gastric gland acquires a mutation. This mutated stem cell would then expand its mutated progeny until it eventually took over the gland and all the cell lineages present would have the mutation. The unit would then spread by gland fission creating a field of mutated gastric glands. A recent study applying this concept to intestinal metaplasia proposed that a gastric stem cell commits to the intestinal phenotype [66]. The progeny of the mutated stem cell would be seen mixed with normal gastric lineages as the stem cell proceeded to take over the gland. This could be one explanation for the mixed glands observed in the $H$. pylori-infected Mongolian gerbils [40]. It is possible that SPEM arises from transdifferentiation of the chief cells, while intestinal metaplasia is derived from a mutated stem cell born in the context of SPEM. Alternatively, intestinal metaplasia may represent a further mucous cell metaplastic differentiation that predisposes to the adoption and propagation of lineages with genetic mutations. If these concepts are correct, then SPEM and intestinal metaplasia may represent sequential steps in the progression to gastric cancer.

\section{Future studies may result in biomarker discovery}

Given the clear association of metaplasias in the stomach with increased risk for gastric cancer, the identification of markers of metaplasia and metaplastic progression to dysplasia is a clear priority for the development of effective screening methods that could identify preneoplasia. The discovery of preneoplastic biomarkers would be of great value for earlier detection and treatment of preneoplastic lesions. Recent studies have begun to identify a number of biomarkers that have implications for cancer outcome. For example, a recent study identified the secreted whey acidic protein (WAP) domain protein, HE4 (WFDC2) as a putative biomarker [35]. While there was no HE4 expression in normal gastric lineages, there was a prominent increase in expression in SPEM lineages from both DMP-777-treated and $H$. felis-infected mice (Fig. 1). Similarly, HE4 was expressed in metaplastic lineages in humans (Fig. 2). Through examination of human tissue arrays, HE4 was found to have strong expression in $100 \%$ of both SPEM and intestinal metaplasias. Additionally, strong HE4 expression is maintained in a number of intestinal-type gastric cancers, making it a promising biomarker candidate. However, a more expansive array of biomarkers is still needed to increase the diagnostic specificity of metaplasias and preneoplastic events. As more biomarkers are established, diagnosis and treatments may be increasingly specialized based on the individual patient's biomarker expression patterns. Additionally, the discovery of new biomarkers will lead to a greater understanding of the molecular progression of normal gastric lineages to metaplasia (SPEM and intestinal metaplasia) and to cancer.

Acknowledgments Dr. Goldenring is supported by grants from a Department of Veterans Affairs Merit Review Award, RO1 DK071590, the AGA Funderburg Award in Gastric Biology Related to Cancer, and support by core resources of the Vanderbilt Digestive Disease Center, P30 DK058404.

\section{References}

1. Pisani P, Parkin DM, Bray F, Ferlay J. Estimates of the worldwide mortality from 25 cancers in 1990. Int J Cancer 1999;83:18-29.

2. Blaser M, Parsonnet J. Parasitism by the "slow" bacterium Helicobacter pylori leads to altered gastric homeostasis and neoplasia. J Clin Invest 1994;94:4-8.

3. Jain RN, Brunkan CS, Chew CS, Samuelson LC. Gene expression profiling of gastrin target genes in parietal cells. Physiol Genomics 2006;24:124-32.

4. Beauchamp RD, Barnard JA, McCutchen CM, Cherner JA, Coffey RJ Jr. Localization of transforming growth factor alpha and its receptor in gastric mucosal cells. J Clin Invest 1989;84: 1017-23.

5. Murayama Y, Miyagawa JI, Higashiyama S, Kondo S, Yabu M, Kanayama S, et al. Localization of heparin-binding epidermal growth factor-like growth factor in human gastric mucosa. Gastroenterology 1995;109:1051-9.

6. Abe S, Sasano H, Katoh K, Ohara S, Arikawa T, Noguchi T, et al. Immunohistochemical studies on EGF family growth factors in normal and ulcerated human gastric mucosa. Dig Dis Sci 1997;42:1199-209.

7. Li Q, Karam SM, Gordon JI. Diphtheria toxin-mediated ablation of parietal cells in the stomach of transgenic mice. J Biol Chem 1996;271:3671-6.

8. El-Zimaity HMT, Ota H, Graham DY, Akamatsu T, Katsuyama T. Patterns of gastric atrophy in intestinal type gastric carcinoma. Cancer 2002;94:1428-36.

9. Correa P. A human model of gastric carcinogenesis. Cancer Res 1988;48:3554-60.

10. Filipe MI, Munoz N, Matko I, Kato I, Pompe-Kirn V, Juersek A, et al. Intestinal metaplasia types and the risk of gastric cancer: a cohort study in Slovenia. Int J Cancer 1994;57:324-9.

11. Hattori T. Development of adenocarcinomas in the stomach. Cancer 1986;57:1528-34.

12. Takizawa T, Koike M. Minute gastric carcinoma from pathomorphological aspect - reconsideration concerning histogenesis of gastric carcinomas. Stomach and Intestine 1998;23:791-800.

13. Hattori T, Fujita S. Tritiated thymidine autotradiographic study on histogenesis and spreading of intestinal metaplasia in human stomach. Pathol Res Pract 1979;164:224-37. 
14. Hattori T, Helpap B, Gedigk P. The morphology and cell kinetics of pseudopyloric glands. Virchows Arch B Cell Pathol Incl Mol Pathol 1982:39:31-40.

15. Xia HH, Kalantar JS, Talley NJ, Wyatt JM, Adams S, Cheung K, et al. Antral-type mucosa in the gastric incisura, body and fundus (antralization): a link between Helicobacter pylori infection and intestinal metaplasia. Am J Gastroenterol 2000;95:114-21.

16. Schmidt PH, Lee JR, Joshi V, Playford RJ, Poulsom R, Wright NA, et al. Identification of a metaplastic cell lineage associated with human gastric adenocarcinoma. Lab Invest 1999;79: 639-46.

17. Yamaguchi H, Goldenring JR, Kaminishi M, Lee JR. Association of spasmolytic polypeptide expressing metaplasia (SPEM) with carcinogen administration and oxyntic atrophy in rats. Lab Invest 2002;82:1045-52.

18. Halldorsdottir AM, Sigurdardottir M, Jonasson JG, Oddsdottir M, Magnusson J, Lee JR, et al. Spasmolytic polypeptide expressing metaplasia (SPEM) associated with gastric cancer in Iceland Dig Dis Sci 2003;48:431-41.

19. Morson BC. Intestinal metaplasia of the gastric mucosa. $\mathrm{Br} \mathrm{J}$ Cancer 1955;9:365-76.

20. Ectors N, Dixon MF. The prognostic value of sulphomucin positive intestinal metaplasia in the development of gastric cancer. Histopathology 1986;10:1271-7.

21. Yamaguchi H, Goldenring JR, Kaminishi.M., Lee JR. Identification of spasmolytic polypeptide expressing metaplasia (SPEM) in remnant gastric cancer and surveillance postgastrectomy biopsies. Dig Dis Sci 2001;47:573-8.

22. Wang TC, Goldenring JR, Dangler C, Ito S, Mueller A, Jeon WK, et al. Mice lacking secretory phospholipase A2 show altered apoptosis and differentiation with Helicobacter felis infection. Gastroenterology 1998;114:675-89.

23. Fox JG, Li X, Cahill RJ, Andrutis K, Rustgi AK, Odze R, et al. Hypertrophic gastropathy in Helicobacter felis-infected wild type C57BL/6 mice and p53 hemizygous transgenic mice. Gastroenterology 1996;110:155-66.

24. Fox JG, Wang TC, Rogers AB, Poutahidis T, Ge Z, Taylor N, et al. Host and microbial constituents influence Helicobacter pylori-induced cancer in a murine model of hypergastrinemia. Gastroenterology 2003;124:1879-90.

25. Nomura S, Yamaguchi H, Wang TC, Lee JR, Goldenring JR. Alterations in gastric mucosal lineages induced by acute oxyntic atrophy in wild type and gastrin deficient mice. Am J Physiol Gastrointest Liver Physiol 2004;288:G362-75.

26. Goldenring JR, Ray GS, Coffey RJ, Meunier PC, Haley PJ, Barnes TB, et al. Reversible drug-induced oxyntic atrophy in rats. Gastroenterology 2000;118:1080-93.

27. Fox JG, Blanco M, Murphy JC, Taylor NS, Lee A, Kabok Z, et al. Local and systemic immune responses in murine Helicobacter felis active chronic gastritis. Infect Immun 1993;61: 2309-15.

28. Karam SM, Leblond CP. Dynamics of epithelial cells in the corpus of the mouse stomach. I. Identification of proliferative cell types and pinpointing of the stem cells. Anat Rec 1993;236: 259-79.

29. Karam SM, Leblond CP. Dynamics of epithelial cells in the corpus of the mouse stomach. II. Outward migration of pit cells. Anat Rec 1993;236:280-96.

30. Karam SM, Leblond CP. Dynamics of epithelial cells in the corpus of the mouse stomach. III. Inward migration of neck cells followed by progressive transformation into zymogenic cells. Anat Rec 1993;236:297-313.

31. Karam SM, Leblond CP. Dynamics of epithelial cells in the corpus of the mouse stomach. V. Behavior of entero-endocrine and caveolated cells: general conclusions of cell kinetics in the oxyntic epithelium. Anat Rec 1993;236:333-40.

32. Karam SM, Straiton T, Hassan WM, Leblond CP. Defining epithelial cell progenitors in the human oxyntic mucosa. Stem Cells 2003;21:322-36.
33. Ramsey VG, Doherty JM, Chen CC, Stappenbeck TS, Konieczny SF, Mills JC. The maturation of mucus-secreting gastric epithelial progenitors into digestive-enzyme secreting zymogenic cells requires Mist1. Development 2007;134:211-22.

34. Nomura S, Baxter S, Yamaguchi T, Leys C, Vartapetian AB, Fox JG, et al. Spasmolytic polypeptide expressing metaplasia (SPEM) to pre-neoplasia in H. felis-infected mice. Gastroenterology 2004; 127:582-94.

35. Nozaki K, Ogawa M, Williams JA, LaFleur BJ, Ng V, Drapkin $\mathrm{RI}$, et al. A molecular signature of gastric metaplasia arising in response to acute parietal cell loss. Gastroenterology 2008: 511-21.

36. Hirayama F, Takagi S, Kusuhara H, Iwao E, Yokoyama Y, Ikeda Y. Induction of gastric ulcer and intestinal metaplasia in Mongolian gerbils infected with Helicobacter pylori. J Gastroenterol 1996;31:755-7.

37. Honda S, Fujioka T, Tokieda M, Gotoh T, Nishizono A, Nasu M. Gastric ulcer, atrophic gastritis, and intestinal metaplasia caused by Helicobacter pylori infection in Mongolian gerbils. Scand J Gastroenterol 1998;33:454-60.

38. Watanabe T, Tada M, Nagai H, Sasaki S, Nakao M. Helicobacter pylori infection induces gastric cancer in Mongolian gerbils. Gastroenterology 1998;115:642-8.

39. Honda S, Fujioka T, Tokieda M, Satoh R, Nishizono A, Nasu M. Development of Helicobacter pylori-induced gastric carcinoma in Mongolian gerbils. Cancer Res 1998;58:4255-9.

40. Yoshizawa N, Takenaka Y, Yamaguchi H, Tetsuya T, Tanaka H, Tatematsu M, et al. Emergence of spasmolytic polypeptideexpressing metaplasia in Mongolian gerbils infected with Helicobacter pylori. Lab Invest 2007;87:1265-76.

41. El-Zimaity HMT, Ramchatesingh J, Saeed MA, Graham DY. Gastric intestinal metaplasia: subtypes and natural history. J Clin Pathol 2001;54:679-83.

42. Sugimura T, Matsukura N, Sato S. Intestinal metaplasia of the stomach as a precancerous stage. IARC Sci Publ 1982:515-30.

43. Lee A, O'Rourke J, De Ungria MC, Robertson B, Daskalopoulos G, Dixon MF. A standardized mouse model of Helicobacter pylori infection: introducing the Sydney strain. Gastroenterology 1997;112:1386-97.

44. Misra V, Misra S, Dwivedi M, Singh UP, Bhargava V, Gupta SC. A topographic study of Helicobacter pylori density, distribution and associated gastritis. J Gastroenterol Hepatol 2000;15:73743.

45. Johnson LR. New aspects of the trophic actions of gastrointestinal hormones. Gastroenterology 1977;72:788-92.

46. Wang TC, Koh TJ, Varro A, Cahill RJ, Dangler CA, Fox JG, et al. Processing and proliferative effects of human progastrin in transgenic mice. J Clin Invest 1996;98:1918-29.

47. Zavros Y, Eaton KA, Kang W, Rathinavelu S, Katukuri V, Kao JY, et al. Chronic gastritis in the hypochlorhydric gastrin-deficient mouse progresses to adenocarcinoma. Oncogene 2005;24:235466.

48. Lefebvre O, Chenard MP, Masson R, Linares J, Dierich A, LeMeur M, et al. Gastric mucosa abnormalities and tumorigenesis in mice lacking pS2 trefoil protein. Science 1996;274:259-62.

49. Judd LM, Alderman BM, Howlett M, Shulkes A, Dow C, Moverley J, et al. Gastric cancer development in mice lacking the SHP2 binding site on the IL-6 family co-receptor gp130. Gastroenterology 2004;126:196-207.

50. Andersson K, Chen D, Mattsson H, Sundler F, Hakanson R. Physiological significance of ECL-cell histamine. Yale J Biol Med 1998;71:183-93.

51. Chen D, Aihara T, Zhao CM, Hakanson R, Okabe S. Differentiation of the gastric mucosa. I. Role of histamine in control of function and integrity of oxyntic mucosa: understanding gastric physiology through disruption of targeted genes. Am J Physiol Gastrointest Liver Physiol 2006;291:G539-44.

52. Hinkle KL, Samuelson LC. Lessons from genetically engineered animal models. III. Lessons learned from gastrin gene deletion in 
mice. Am J Physiol Gastrointest Liver Physiol 1999;277:G5005.

53. Jain RN, Samuelson LC. Differentiation of the gastric mucosa. II. Role of gastrin in gastric epithelial cell proliferation and maturation. Am J Physiol Gastrointest Liver Physiol 2006;291:G7625.

54. Lindstrom E, Chen D, Norlen P, Andersson K, Hakanson R. Control of gastric acid secretion: the gastrin-ECL cell-parietal cell axis. Comp Biochem Physiol A Mol Integr Physiol 2001;128: 505-14.

55. Samuelson LC, Hinkle KL. Insights into the regulation of gastric acid secretion through analysis of genetically engineered mice. Annu Rev Physiol 2003;65:383-400.

56. Zimmerhackl B, Wunsch E, Classen M, Schusdziarra V, Schepp W. In man histamine and muscarinergic mechanisms are essential mediators of acid secretion in response to synthetic human gastrin (1-17). Regul Pept 1993;23:583-92.

57. Nozaki K, Weis V, Wang TC, Falus A, Goldenring JR. Altered gastric chief cell lineage differentiation in histamine-deficient mice. Am J Physiol Gastrointest Liver Physiol 2009;296:G121120.

58. Chew CS. Inhibitory action of somatostatin on isolated gastric glands and parietal cells. Am J Physiol Gastrointest Liver Physiol 1983;245:G221-9.

59. Ogawa M, Nomura S, Varro A, Wang TC, Goldenring JR. Altered metaplastic response of waved-2 EGF receptor mutant mice to acute oxyntic atrophy. Am J Physiol Gastrointest Liver Physiol 2006;290:G793-804.

60. Nam KT, Varro A, Coffey RJ, Goldenring JR. Potentiation of oxyntic atrophy-induced gastric metaplasia in amphiregulindeficient mice. Gastroenterology 2007;132:1804-19.

61. Wang TC, Dangler CA, Chen D, Goldenring JR, Koh T, Raychowdhury R, et al. Synergistic interaction between hypergastrinemia and Helicobacter infection in a mouse model of gastric cancer. Gastroenterology 2000;118:36-47.

62. Mohammadi M, Czinn S, Redline R, Nedrud J. Helicobacterspecific cell-mediated immune responses display a predominant Th1 phenotype and promote a delayed-type hypersensitivity response in the stomachs of mice. J Immunol 1996;156:4729-38.

63. Roth KA, Kapadia SB, Martin SM, Lorenz RG. Cellular immune responses are essential for the development of Helicobacter felisassociated gastric pathology. J Immunol 1999;163:1490-7.

64. Houghton J, Stoicov C, Nomura S, Carlson J, Li H, Rogers AB, et al. Gastric cancer originating from bone marrow derived cells. Science 2004;306:1568-71.

65. Slaughter DP, Southwick HW, Smejkal W. Field cancerization in oral stratified squamous epithelium; clinical implications of multicentric origin. Cancer 1953;6:963-8.

66. McDonald SA, Greaves LC, Gutierrez-Gonzalez L, RodriguezJusto M, Deheragoda M, Leedham SJ, et al. Mechanisms of field cancerization in the human stomach: the expansion and spread of mutated gastric stem cells. Gastroenterology 2008;134:500-10. 